\title{
Factores que inciden en la gestión de proyectos de investigación científica
}

Marco Aurelio Rosario Villarreal ${ }^{1 \mathrm{a}}$, Yolvi Ocaña Fernández ${ }^{2}$, Cesar Herminio Capillo Chávez3, Alejandrino Benito Lavado Rodríguez ${ }^{4}$, Mohammed El Homrani ${ }^{5}$ y S. Mateo Arias Romero $^{6}$

\author{
Universidad Nacional de Huancavelica, Huancavelica, Perú ${ }^{1}$ \\ Universidad César Vallejo, Lima, Perú ${ }^{234}$ \\ Universidad de Granada, Granada, España ${ }^{56}$ \\ Orcid ID: https://orcid.org/0000-0003-0250-9137 \\ Orcid ID: https://orcid.org/0000-0002-2566-6875 \\ Orcid ID: https://orcid.org/0000-0002-2765-47203 \\ Orcid ID: https://orcid.org/0000-0002-6717-051X ${ }^{4}$ \\ Orcid ID: https://orcid.org/0000-0003-3051-4101 \\ Orcid ID: https://orcid.org/0000-0002-2152-2153 ${ }^{6}$
}

\section{Recibido: 08 de marzo de 2018 Aceptado: 15 de noviembre de 2018}

\section{Resumen}

La presente investigación pretende explicar que existen factores externos, que influyen directamente en la gestión de los proyectos de investigación científica y tecnológica financiados con recursos del Fondo de desarrollo socioeconómico de Camisea (Focam) de la Universidad Nacional de Huancavelica (UNH). Dentro de estos factores externos que inciden directamente se encuentran las competencias profesionales de los docentes investigadores y la gestión logística que permite la implementación de la investigación. Se utiliza el enfoque cuantitativo, el tipo de investigación sustantiva explicativa y el diseño de investigación es no experimental de corte transversal tipo explicativo causal. La muestra censal con un tamaño de muestra de 49 docentes investigadores de Ingeniería. Para la presente investigación, se usó la técnica de la encuesta para las tres variables de estudio. Se puede afirmar que existe influencia positiva de las competencias profesionales y la gestión logística sobre la gestión de los proyectos de investigación científica financiados con recursos de Focam. Es la primera investigación que 
ofrece una discusión sobre en qué nivel de gestión se encuentran los proyectos de investigación científica ejecutados en la Universidad Nacional de Huancavelica, desde la planificación de la investigación hasta la publicación del artículo científico en una revista indizada. Se plantean alternativas de solución para los investigadores y para la oficina de logística que ayudarán a gestionar eficientemente los proyectos de investigación científica.

Palabras claves: planificación de proyectos, gestión de proyectos, competencias profesionales, gestión logística.

\title{
Factors that affect the management of scientific research projects
}

\begin{abstract}
This research study aims to explain how there are external factors that directly influence the management of scientific and technological research projects financed with resources from the socioeconomic development fund of Camisea (Focam) of the National University of Huancavelica (UNH). Within these external factors that directly affect are the professional competencies of the research faculty and the logistic management that allows for the implementation of the research projects. A quantitative approach is used for this explanatory substantive research and the research design is non-experimental, cross-sectional, explanatory and causal. The sample included 49 engineering research profesors. For the present investigation, the survey technique was used for the three study variables. Findings - It can be affirmed that there is a positive influence of the professional competences and the logistic management on the management of the scientific research projects financed with resources of Focam of the UNH. This is the first research study that offers a discussion on the level of management of the scientific research projects carried out at the National University of Huancavelica, from research planning to the publication of the scientific article in an indexed journal. Alternative solutions are proposed for researchers and the logistics office to help efficiently manage scientific research projects.
\end{abstract}

Keywords: project planning, project management, professional skills, logistics management. 


\section{Introducción}

Los institutos y las unidades de investigación de la Universidad Nacional de Huancavelica (UNH), se encargan del desarrollo y la gestión de la investigación científica y tecnológica como una función principal de la universidad a través de la ejecución de los proyectos de investigación, con la finalidad de atender y solucionar las necesidades de la sociedad especialmente del ámbito regional y nacional (Asamblea Estatutaria UNH 2014).

En la UNH desde el año 2007, los docentes ejecutan proyectos de investigación científica y tecnológica financiada con recursos de Focam. Hasta el 2015, La Dirección Universitaria de Investigación (DUI) era la encargada de gestionar y monitorear el financiamiento y la ejecución de los proyectos de Focam. A partir del año 2016, son los Institutos de investigación quienes asumen esta función de gestión de los proyectos.

Independientemente de la universidad en la que nos encontremos, Estrada, Quiñónez y Pantoja (2017) señalan que es tarea de las universidades generar conocimientos nuevos sin dedicarse exclusivamente a reproducir lo que ya existe, por lo que el profesorado que desarrolla su labor en los centros universitarios debe contar con competencias al respecto. Señalan, además, que es una exigencia para los investigadores la capacidad de analizar y cuestionar información, además de argumentar sus juicios. Llegados a este punto, podemos también incluir, entre las competencias que debe disponer como investigador, la de la gestión de proyectos de investigación.

Una parte de la gestión de los proyectos aprobados es la implementación de los mismos, que involucra principalmente a las siguientes oficinas administrativas de la institución: la oficina de planificación y presupuesto para aprobar y presupuestar los gastos por mes; y a la oficina de Logística para la adquisición de bienes y servicios requeridos por los proyectos de Focam. Pero la gestión de los proyectos en estas oficinas administrativas no es eficiente, debido al incumplimiento y retrasos de los procedimientos que deben seguirse de acuerdo a las directivas establecidas para implementar los proyectos de investigación, resultando en reprogramaciones del proyecto en más tiempo de lo establecido.

Otra parte de la gestión de los proyectos aprobados consiste en la planificación y la ejecución de los proyectos de investigación, que involucra a los mismos investigadores. Pero de acuerdo a los planes de los proyectos de investigación de ingeniería, no hay una coherencia entre los objetivos de la investigación y los avances trimestrales presentados durante la ejecución de los proyectos, produciéndose el incumplimiento de los objetivos y resultados finales de la investigación con la calidad y plazos establecidos. 
Por tanto, la presente investigación pretende evaluar el nivel de gestión de los proyectos del Instituto de Ingeniería, con la finalidad de conocer y explicar los factores o causas más preponderantes que inciden directamente en la gestión de los proyectos de Focam, y finalmente plantear alternativas de solución que ayuden a cumplir los objetivos de la investigación y obtener los resultados esperados. Según los expertos del tema de investigación, existen dos factores principales que afectan en la gestión del proyecto de investigación: las competencias profesionales de los investigadores y la gestión logística de los proyectos. Entonces, se ha realizado el estudio en base a estos dos factores mencionados para evaluar el nivel de gestión de los proyectos de Focam.

La gestión de proyectos globales implica proyectos de alta complejidad, en los que sería difícil la gestión utilizando enfoques tradicionales (orientado a actividades y tareas). Esta investigación demuestra que los principales retos de organización en proyectos globales son: los gerentes de proyecto y los actores externos en el proyecto, y se concluye que las empresas necesitan un enfoque de gestión de basado en relaciones para gestionar estos retos en proyectos globales (Aarseth, Rolstadas y Andersen, 2013).

Los esfuerzos de los investigadores en temas de gestión de proyectos, deben estar dirigidos a desarrollar modelos prácticos de gestión de proyectos (PM) que ayuden a los profesionales que desarrollan proyectos a mejorar su capacidad para entregar proyectos de manera eficiente y optimizar de manera efectiva los beneficios; esto requiere gestionar la definición del proyecto, así como la ejecución del proyecto (Morris, 2010).

Las características potenciales de liderazgo en la ejecución de proyectos, sería la base para desarrollar estrategias y criterios personalizados para seleccionar los directores de proyectos de investigación y desarrollo con habilidades y comportamientos adecuados. El estudio ha encontrado una serie de características potenciales que los directores de $\mathrm{I}+\mathrm{D}$ deben tener, como son: la creatividad, el razonamiento y el aprendizaje, aceptación de riesgos y fallas, atributos analíticos y de originalidad, enfoque realista y objetivo, entre otros (Ram y Ronggui, 2018).

El uso de procesos y herramientas de gestión de proyectos permite que un proyecto de investigación cumpla con mayor probabilidad sus objetivos (Rodríguez y Caurcel, 2019) dentro de los parámetros de alcance, tempo, costo y calidad. Además, se sugiere la capacitación en gestión de proyectos por parte de los profesores, ampliar los servicios de apoyo a la investigación disponibles para los profesores, y procesos sistemáticos para involucrar a los profesores asistentes en servicios de apoyo a la investigación, con la finalidad de transformar 
las prácticas de gestión de proyectos de investigación de los profesores (Alpert y Hartshorne, 2013).

El éxito de la gestión de proyectos aplicada a un proyecto caso de salud, requiere de procedimientos formales como los diversos procesos (inicio, planeación, ejecución, seguimiento y cierre) y clases de conocimiento establecidos en base a la metodología del PMI (Instituto de Gestión de Proyectos), y que estos proyectos deben convertirse en modelos debidamente formalizados e incluidos en una base de datos para que sean consultadas por los gestores de futuros proyectos (Toro y Bernate, 2013).

El PMI es un modelo de planificación y gestión de proyectos de desarrollo del sector productivo para la obtención específicamente de productos cumpliendo con la calidad esperada. Pero también se puede aplicar a otros sectores de la sociedad, pero es necesario adaptarlo a los requerimientos que exige el proyecto en particular. Este modelo se basa en la aplicación de 9 áreas de conocimientos, de habilidades, de herramientas y de técnicas durante el ciclo de vida del proyecto. Considera dos dimensiones: la planificación y la gestión del proyecto. La gestión del proyecto está más relacionada con las actividades de asignación de recursos, formación de equipos, desarrollo, pruebas y evaluación de resultados (Fernandes y Guimarães, 2013).

El modelo de gestión de proyectos GDPM está orientado más a los proyectos de negocio, donde se requieren cambios de actitudes del personal directivo y técnico y cambios en los procesos de transformaciones de las organizaciones de negocio. La planificación y gestión de actividades está enfocada a la aplicación de habilidades, técnicas y herramientas apropiadas de la disciplina de cada proyecto por parte del personal directivo y técnico para cumplir con las actividades planificadas. El control y monitoreo de los avances de las actividades se realiza a través del plan de hitos del modelo GDPM y el control de las responsabilidades de los actores involucrados en el proyecto, se realiza a través del instrumento de gestión matriz de responsabilidades (Ramón, García y Lamarca, 2013).

"Skopos" es un modelo de gestión de proyectos educativos centrados en el concepto. Está organizado en tres partes principales: el concepto, plan de acción y plan de control y evaluación, siendo el concepto la base para la elaboración de los planes de acción y de control. Básicamente este modelo se centra en la planificación del proyecto educativo donde se detallan los objetivos, los resultados deseados, las actividades y tareas, los costos y recursos necesarios, el cronograma del proyecto, la matriz de control de productos y resultados, análisis de riesgos, etc. (Fernandes y Guimarães, 2013).

Las 5 fases del ciclo de vida de un proyecto informático son las siguientes y se realizan las tareas que se explicitan (Ramón, García y Lamarca, 2013): 
- En la fase de Aprobación del proyecto se analiza la viabilidad técnica y económica y se aprueba el proyecto.

- En la fase de Definición del proyecto se detallan los objetivos que se desean alcanzar, una definición de los recursos y la identificación de los riesgos.

- En la fase de Planificación se detalla la distribución de las actividades y tareas a realizar, se detalla el calendario de hitos y la distribución de los recursos.

- En la fase de Ejecución se ejecutan y se verifican los avances de las actividades del proyecto, se realiza gestión de riesgos durante esta fase.

- En la fase de Cierre se realizan las pruebas del rendimiento del sistema y la verificación por parte de los usuarios. Se entrega el informe final del proyecto y los planes de mantenimiento preventivo y correctivo del sistema.

Las competencias profesionales del profesional de ingeniería es la aplicación de los conocimientos, habilidades y actitudes desarrolladas durante la formación en situaciones del ejercicio profesional como es la gestión de los proyectos de investigación científica y tecnológica. Pero también las competencias profesionales se refieren a las funciones y roles de un profesional que deben cumplir para desempeñar en forma adecuada e idónea su labor profesional (Gómez, 2015).

Se pueden predecir el desempeño profesional o una gestión en un contexto laboral, por medio de la medición de los conocimientos, habilidades, actitudes y valores adquiridos durante la formación profesional y aplicada a la labor profesional que desempeña. Estas son las competencias profesionales (Arroyo, 2012).

Cuando un profesional domina los conocimientos y destrezas necesarios para su labor profesional, se denomina competencia técnica. Y cuando aplica esos conocimientos y destrezas para cumplir adecuadamente con las funciones y roles de su labor profesional se denominan competencia metodológica. Ambas competencias se suman para formar las competencias duras (Gómez, 2015).

Las competencias duras son los conocimientos y destrezas adquiridas sobre un tema específico que permite que el profesional cumpla con los roles que demanda la labor profesional en su entorno de trabajo (Conexión Esan, 2015).

Cuando un profesional sabe colaborar con otras personas en forma constructiva y muestra un comportamiento orientado al grupo de trabajo, se denomina competencia social. Y cuando aplica estas actitudes y valores en su entorno de trabajo, es capaz de organizar, decidir 
y aceptar responsabilidades, se denomina competencia participativa. Ambos sumados forman las competencias blandas (Gómez, 2015).

Para contratar un profesional para un puesto de trabajo, la oficina de recursos humanos de la organización elabora un perfil donde se señala no solamente las competencias duras, sino también las competencias blandas requeridas para el puesto. Estas habilidades blandas son las marcan la verdadera diferencia entre los postulantes al puesto. Estas habilidades blandas están asociadas al comportamiento de la persona, su desempeño social, liderazgo y manejo emocional (Conexión Esan, 2015).

La gestión logística relacionada con la velocidad del flujo logístico para proveer de bienes y servicios a las unidades o áreas de una organización, con la finalidad de que puedan cumplir con sus funciones y tareas en los plazos establecidos. Esto permitirá medir el desempeño de estas áreas funcionales. Se puede establecer tres indicadores para medir la gestión logística: la velocidad del flujo logístico, desde el momento que se genera el pedido hasta que se adquiera y se entregue a los usuarios finales. Esto permite identificar y controlar los cuellos de botella. El segundo indicador está relacionado con los costos que se agregan a los bienes y servicios requeridos durante el flujo logístico. Y el último indicador está relacionado con el grado de satisfacción de los usuarios finales con respecto a la gestión logística de la organización (Villegas, 2006).

La gestión logística es el encargado de realizar el abastecimiento oportuno de los requerimientos de bienes y servicios en general, incluidos los requeridos por los proyectos de investigación científica financiada con recursos de focam en función al cronograma de actividades y presupuesto asignado, cumpliendo para ello una serie de procesos y procedimientos para la contracción de bienes y servicios (UNH, 2016a).

La ley de contrataciones del Estado $\mathrm{N}^{\circ}$ 30225, establece las disposiciones y lineamientos que deben seguir las entidades del sector público para la contratación oportuna de bienes y servicios con las mejores condiciones de precio y calidad, con la finalidad de lograr los fines públicos y garantizar la satisfacción de los usuarios finales. La gestión logística está asociada principalmente a los dos primeros títulos. Título I, relacionado con los responsables de las contrataciones del estado y la calificación de los proveedores. Título II, relacionado con el proceso de contratación desde el requerimiento de contratación por parte del usuario final hasta la ejecución del proceso de selección establecido. Se establece el método de contratación en base al valor estimado de las contrataciones de bienes y valor referencial de las contrataciones de servicios (OSCE-L, 2014). 
El reglamento de la ley de contrataciones del Estado $\mathrm{N}^{\circ} 30225$, permite normar los procesos y procedimientos utilizados para la contratación de bienes y servicios por parte de las instituciones del sector público. La gestión logística está asociada principalmente a los cuatro primeros títulos:

- Título I, se encarga de normar la organización encargada de la gestión administrativa de las contrataciones de bienes y servicios del estado.

- Título II, encargado de normar la planificación de las contrataciones de bienes y servicios contenidas en el plan anual de adquisiciones, en cuanto a su formulación, aprobación y modificación.

- Título III, se encarga de normar el requerimiento de contratación, la preparación y aprobación del expediente técnico para convocar a un proceso de selección. Incluye las normas para la preparación, conducción y realización del proceso de selección a cargo de un comité de selección u órgano de contratación. Noema los documentos necesarios para el proceso de selección como las bases de la convocatoria y la solicitud de cotización.

- Y el título IV, encargado de normar las etapas de las convocatorias de los procesos de selección establecidos (OSCE-R, 2014).

El proceso de contratación de bienes y servicios pasa por una serie de etapas y procedimientos.

- Primero: es responsabilidad del área usuaria verificar la programación de las necesidades de bienes y servicios en el cuadro de necesidades, o en el plan operativo institucional o en cuadro presupuestal de gastos de los proyectos de focam.

- Segundo: el área usuaria realiza la formulación del requerimiento de bienes y servicios (especificaciones técnicas y términos de referencia) en el primer trimestre de cada ejercicio fiscal.

- Tercero: el área usuaria ejecuta el requerimiento de contratación de bienes y servicios a través del pedido de compra ingresado al sistema SIGA y presentado en físico a la oficina de logística, adjuntando los formatos de especificaciones técnicas y términos de referencia. 
- Cuarto: La oficina de logística lleva a cabo el proceso de selección (licitaciones y concursos públicos, adjudicación simplificada, contratación directa, compras directas, etc.) respetando los plazos promedios de ejecución.

- Quinto: los bienes adquiridos son internados en el almacén para su verificación cuantitativa y cualitativa y conformidad respectiva. Para los servicios contratados, el producto del servicio debe ser presentado mediante un informe completo del proveedor al área usuaria.

- Y sexto: la conformidad de los bienes está a cargo del área técnica especialista en la materia. El área usuaria es la encargada de dar la conformidad del servicio a través del sistema SIGA. Para servicios especializados, el área técnica especialista en la materia es la que otorga la conformidad respectiva (UNH, 2016b).

El proceso de selección por compras directas para contratación de bienes y servicios menores o iguales a 8 UIT, la gestión logística se realiza como sigue:

- Primero, el área usuaria realiza el requerimiento de contratación en el sistema SIGA, detallando en forma clara y precisa las especificaciones técnicas de los bienes y los términos de referencia de los servicios.

- Segundo, la unidad de abastecimiento realiza el estudio de mercado para obtener hasta tres cotizaciones para pluralidad de postores y marcas. Y son admitidas aquellas cotizaciones que cumplan con las condiciones de calidad requeridas, para elaborar el cuadro comparativo correspondiente y conocer el proveedor ganador.

- Tercero, la oficina de presupuesto se encarga de emitir la certificación de crédito presupuestario que garantiza la disponibilidad de recursos y la fuente de financiamiento.

- Cuarto, la unidad de abastecimiento se encarga de generar las órdenes de compra y ordenes de servicio con las penalidades correspondientes, así como el registro de las órdenes en el sistema SEACE. La unidad de adquisición se encarga de registrar el compromiso en el sistema SIAF.

- Quinto, el área de trámite documentario se encarga de notificar y entregar oportunamente las órdenes a los proveedores ganadores. Sexto, el área de 
almacén realiza la recepción y verificación de los bienes adquiridos y la entrega a los usuarios finales (UNH, 2016a).

La gestión de la investigación científica se realiza en proyectos y es un desarrollo específico que se inicia con la idea de una pregunta o problema que se quiere dar respuesta o solución. Se planifica la idea en el plan del proyecto, tras revisión del estado del arte (Gallego y Rodríguez, 2015), se realiza la implementación, la organización y la ejecución del proyecto, terminando en la documentación y publicación de los resultados. Y para que tenga la opción de ser evaluado y financiado con recursos externos, debe contener 3 elementos principales y ser consistentes entre sí (Bauer, Bleck-Neuhaus y Dombois, 2010):

- Un planteamiento científico del problema y los objetivos claramente definidos;

- Un diseño de investigación transparente y realista;

- Y un plan de acción detallando los productos esperados como la publicación y plan de uso de los recursos:

○ Si el diseño metodológico es el adecuado para el planteamiento del problema;

- Si el diseño metodológico es preciso para alcanzar los objetivos,

- Si los métodos científicos son los adecuados al cronograma y al presupuesto;

- Si la propuesta es innovadora y de utilidad científica y social.

El desarrollo de la investigación científica y tecnológica pasa por las etapas del planeamiento, evaluación, la organización, la implementación, la ejecución, la evaluación y la comunicación de la investigación (Rodríguez, 2005). En el planeamiento se diseña, se formula y se elabora la pertinencia y viabilidad del plan de investigación: se define el planteamiento del problema, los objetivos de la investigación, el estado del arte del tema a estudiar, las hipótesis, la metodología a emplear, el plan de actividades y el plan de recursos, el cronograma y el presupuesto (Carrasco, 2013).

Para la evaluación del proyecto de investigación científica se toma en cuenta tres aspectos principales como son (RegFocamDUI, 2012):

- La trayectoria investigativa de los miembros del equipo de trabajo;

- Las cualidades de la propuesta de investigación: la originalidad, relevancia e impacto esperado en la sociedad; 
- Y la formulación del proyecto de investigación como la calidad de la metodología empleada, la consistencia interna entre el planteamiento del problema, objetivos e hipótesis, coherencia entre presupuesto, cronograma, objetivos y metodología empleada.

La organización es un factor clave en la investigación y tiene que ver con los siguientes puntos (Bauer, Bleck-Neuhaus y Dombois, 2010):

- Las tareas, responsabilidades y la división del trabajo entre los colaboradores;

- Lo anterior es la base para buscar y seleccionar los colaboradores con la especialidad adecuada a través de convocatorias o recomendaciones de colegas investigadores;

- La inducción al nuevo personal sobre la investigación y los resultados esperados,

- Y la cooperación en el proyecto sobre las formas de comunicación, de discusión y manejo de los diferentes tipos de conflictos surgidos en el grupo.

Para implementar la investigación, es necesario contar con los recursos materiales y equipos para acondicionar la oficina de trabajo, es contar con los recursos económicos para gastos de viáticos y pasajes para estudios de campo, es contar con instrumentales y equipos para la recolección y procesamiento de los datos obtenidos en campo, y la realización de la capacitación especializada al equipo de trabajo que participará en la investigación, con la finalidad de poder ejecutar el plan de investigación (Carrasco, 2013).

La ejecución de la investigación es la puesta en marcha del plan de investigación, aplicando los instrumentos diseñados para la recolección de datos, el procesamiento y análisis de la información con la finalidad de lograr los objetivos previstos. Con frecuencia es recomendable aplicar una prueba piloto del instrumento para verificar el funcionamiento del proceso de recolección de datos. Se pueden presentar errores en el procesamiento y análisis de los datos, debido al instrumento mal diseñado o la aplicación de procedimientos estadísticos inadecuados (Bauer, Bleck-Neuhaus y Dombois, 2010).

En la fase de control y seguimiento de la investigación, se debe monitorizar la salud del proyecto con respecto a los parámetros de tiempo, recursos y resultados esperados. El control y seguimiento en el ámbito estratégico, se relaciona con el cumplimiento del plan de hitos o plan de resultados con la calidad esperada y las responsabilidades del equipo de trabajo. El control y seguimiento en el ámbito operativo, se relaciona con el cumplimiento del paquete de actividades y tareas asignadas a los miembros del equipo en los lapsos de tiempo programados. 
En caso de retrasos durante la ejecución del proyecto se debe modificar en consecuencia el plan y el cronograma de actividades (Ramón, García y Lamarca, 2013).

El cierre de la investigación se refiere a la presentación del informe final de investigación de acuerdo a un formato establecido, después de concluir el cronograma de actividades del proyecto de investigación. Sera remitido a la comisión de evaluación para su revisión y aprobación bajo un instrumento de evaluación que será de conocimiento por los investigadores, en el plazo previsto por el reglamento de focam (RegFocamDUI, 2012).

Una vez aprobado el informe final, se debe divulgar el producto de la investigación (resultados obtenidos) a través de la publicación de un artículo científico en una revista indizada a nivel nacional o internacional. La realización de eventos académicos como seminarios, conferencias, cursos y talleres debe considerarse como un medio de divulgación de los resultados de la investigación (Fernandes y Guimarães, 2013).

Se ha formulado el siguiente problema general: ¿Cómo influyen las competencias profesionales y la gestión logística en la gestión de los proyectos de investigación científica financiados con recursos de focam de la Universidad Nacional de Huancavelica? Y los siguientes problemas específicos: ¿Cómo influyen las competencias profesionales y la gestión logística en cada una de las dimensiones de la gestión de los proyectos de investigación científica, es decir: en el planeamiento, en la evaluación y aprobación del proyecto, en la organización, en la implementación, en la ejecución, en el control y seguimiento, ¿en el cierre y en la comunicación de la investigación?

Ante tales interrogantes, se ha planteado el siguiente objetivo general: Demostrar que las competencias profesionales y la gestión logística influyen positivamente en la gestión de los proyectos de investigación científica financiados con recursos de focam de la Universidad Nacional de Huancavelica. Y los siguientes objetivos específicos: Demostrar que las competencias profesionales y la gestión logística influyen positivamente en cada una de las dimensiones de la gestión de los proyectos de investigación científica, es decir: en el planeamiento, en la evaluación y aprobación del proyecto, en la organización, en la implementación, en la ejecución, en el control y seguimiento, en el cierre y en la comunicación de la investigación.

\section{Metodología}

El presente estudio de investigación emplea un diseño no experimental, transversal, tipo explicativo causal. La primera variable son las competencias profesionales, la segunda variable es la gestión logística y la tercera variable es la gestión de los proyectos de investigación 
científica financiados con recursos de Focam. Habiendo definido conceptualmente las tres variables, pasamos a definir operacionalmente las variables.

Las competencias profesionales constan de dos dimensiones: a) las competencias duras y blandas, 2 indicadores y 14 ítems; fueron medidas a través del tipo escala de Likert; b) la gestión logística consta de 7 dimensiones: requerimiento de contratación, cotización y cuadro comparativo, adquisición, proceso de selección y adjudicación, proveedores y penalidades, recepción y almacenamiento y distribución, 13 indicadores y 58 ítems; fueron medidas a través del tipo escala de Likert. La gestión de los proyectos de investigación científica financiada con recursos de Focam consta de 8 dimensiones: planeamiento, evaluación y aprobación, organización, implementación, ejecución, control y seguimiento, cierre y comunicación de la investigación, 36 indicadores y 114 ítems; fueron medidas a través del tipo escala de Likert.

La población de estudio está conformada por todos los docentes investigadores responsables y miembros que han ejecutado proyectos de investigación científica y tecnológica financiados con recursos de Focam del Instituto de Investigación de Ingeniería de la Universidad Nacional de Huancavelica que ascienden a un total de 49 investigadores. Por tratarse de una población finita y accesible, se toma la decisión de utilizar una muestra censal para la presente investigación, es decir, los 49 docentes investigadores que conforman la población van a ser tomados en la muestra.

Para la presente investigación, se utilizó la encuesta como técnica de recolección de datos y como instrumento el cuestionario tipo respuesta directa. En los cuestionarios se establecieron los ítems que deben de medir los indicadores relacionados con las dimensiones de las variables de estudio. Para ello, se diseña tres tipos de encuestas: cuestionario de las competencias profesionales, cuestionario de la gestión logística y cuestionario de la gestión de los proyectos de investigación científica financiada con recursos de Focam.

Se ha realizado la validación de contenido de los instrumentos de recolección de datos a través de juicio de expertos: para el instrumento que mide la variable 1: las competencias profesionales, se ha obtenido un índice de validez de contenido (IVC) de 1.00 y un nivel de significación estadística (p-valor) de 0.031 para cada ítem respectivo; para el instrumento que mide la variable 2: la gestión logística, se ha obtenido un índice de validez de contenido (IVC) de 0.980 y un nivel de significación estadística (p-valor) de 0.031 para cada ítem respectivo; para el instrumento que mide la variable 3: la gestión de los proyectos de investigación científica financiada con recursos de focam, se ha obtenido un índice de validez de contenido (IVC) de 1.00 y un nivel de significación estadística (p-valor) de 0.031 para cada ítem 
respectivo. Para la validez de constructo de los instrumentos se ha obtenido la varianza total explicada y la matriz de componentes rotados para las tres variables de estudio.

Se ha determinado la confiabilidad de los instrumentos de recolección de datos mediante el cálculo del coeficiente alfa de Cronbach. Para el instrumento que mide la variable 1: las competencias profesionales, se ha obtenido un coeficiente alfa de Cronbach de 0.911; para el instrumento que mide la variable 2: la gestión logística, se ha obtenido un coeficiente alfa de Cronbach de 0.969; para el instrumento que mide la variable 3: la gestión de los proyectos de investigación científica financiada con recursos de focam, se ha obtenido un coeficiente alfa de Cronbach de 0.875. Como los datos son no paramétricos, se utilizará la estadística no paramétrica (regresión logística nominal).

\section{Resultados}

A nivel de la variable 1 (competencias profesionales), más del 50\% de los profesionales que realizan proyectos de investigación científica financiada con recursos de Focam, presentan un nivel bajo de competencias profesionales, un $30 \%$ presenta un nivel regular y solo un $20 \%$ presentan un nivel alto de competencias profesionales. Se puede concluir que las competencias profesionales presentan una tendencia de nivel bajo.

A nivel de las dimensiones de la variable 1, a nivel de competencias duras más del 50\% de los profesionales que realizan proyectos de investigación científica financiada con recursos de Focam, presentan un nivel bajo de competencias duras, un $24 \%$ presentan un nivel regular y solo un $20 \%$ presentan un nivel alto de competencias duras. A nivel de competencias blandas, cerca del 50\% de los profesionales presentan un nivel bajo de competencias blandas, un 30\% presentan un nivel regular y solo un $20 \%$ presentan un nivel alto de competencias blandas.

A nivel de la variable 2 (gestión logística), más del $71 \%$ de los profesionales que realizan proyectos de investigación científica financiada con recursos de Focam, perciben un nivel bajo de gestión logística de sus proyectos, y solo un $28 \%$ perciben una gestión logística regular de sus proyectos. Se puede concluir que la gestión logística presenta una tendencia de nivel bajo.

A nivel de las dimensiones de la variable 2, a nivel de requerimiento de contratación más del 53\% de los profesionales que realizan proyectos de investigación científica financiada con recursos de Focam, perciben un nivel bajo de gestión logística, un 30\% percibe un nivel regular y solo un $16 \%$ percibe un nivel alto de gestión logística; a nivel de cotización y cuadro comparativo más del $40 \%$ perciben un nivel bajo de gestión logística, un $47 \%$ percibe un nivel regular y solo un $12 \%$ percibe un nivel alto de gestión logística; a nivel de adquisición más del $63 \%$ perciben un nivel bajo de gestión logística, un $28 \%$ percibe un nivel regular y solo un $8 \%$ 
percibe un nivel alto de gestión logística; a nivel de selección y adjudicación más del 71\% perciben un nivel bajo de gestión logística, un $16 \%$ percibe un nivel regular y solo un $12 \%$ percibe un nivel alto de gestión logística; a nivel de proveedores y penalidades más del $40 \%$ perciben un nivel bajo de gestión logística, un $16 \%$ percibe un nivel regular y un $43 \%$ percibe un nivel alto de gestión logística; a nivel de recepción y almacenamiento más del $40 \%$ perciben un nivel bajo de gestión logística, un $45 \%$ percibe un nivel regular y solo un $14 \%$ percibe un nivel alto de gestión logística; y a nivel de distribución más del 67\% perciben un nivel bajo de gestión logística, un 27\% percibe un nivel regular y solo un 6\% percibe un nivel alto de gestión logística.

A nivel de la variable 3 (gestión de los proyectos de investigación científica y tecnológica), más del $50 \%$ de los profesionales que realizan proyectos de investigación científica financiada con recursos de focam, presentan un nivel bajo de gestión de su proyecto de investigación científica, un $29 \%$ presentan un nivel regular y solo un $20 \%$ presentan una buena gestión de su proyecto de investigación. Se puede concluir que la gestión de los proyectos de investigación científica presenta una tendencia de nivel bajo. Los resultados obtenidos a nivel de las dimensiones de la variable 3 son:

- En el planeamiento de la investigación más del $37 \%$ de los profesionales que realizan proyectos de investigación científica financiada con recursos de focam, presentan un nivel bajo de gestión de su proyecto de investigación y un 63\% presenta un nivel regular de gestión;

- En la evaluación y aprobación de la investigación más del 47\% presenta un nivel bajo de gestión de su proyecto, un $47 \%$ presenta un nivel regular y solo un $6 \%$ presenta un nivel alto de gestión de su proyecto;

- En la organización de la investigación más del $51 \%$ presenta un nivel bajo de gestión de su proyecto y un 49\% presenta un nivel regular de gestión;

- En la implementación de la investigación más del $45 \%$ presenta un nivel bajo de gestión de su proyecto, un $27 \%$ presenta un nivel regular y solo un $28 \%$ presenta un nivel alto de gestión de su proyecto;

- En la ejecución de la investigación más del 65\% presenta un nivel bajo de gestión de su proyecto, un $10 \%$ presenta un nivel regular y un $25 \%$ presenta un nivel alto de gestión de su proyecto; 
- En el seguimiento y control de la investigación más del $16 \%$ presenta un nivel bajo de gestión de su proyecto, un $61 \%$ presenta un nivel regular y solo un $22 \%$ presenta un nivel alto de gestión de su proyecto;

- En el cierre de la investigación más del $45 \%$ presenta un nivel bajo de gestión de su proyecto, un $20 \%$ presenta un nivel regular y un $35 \%$ presenta un nivel alto de gestión de su proyecto;

- En la comunicación de la investigación más del 37\% presenta un nivel bajo de gestión de su proyecto y un $63 \%$ presenta un nivel regular de gestión de su proyecto.

Para la prueba de la hipótesis general, de la información de ajuste de la gestión de los proyectos de investigación mostrada por SPSS la significancia es 0,000 menor a 0,05, en consecuencia se rechaza la hipótesis nula (Ho) a un nivel de confianza del 95\% y se acepta que las competencias profesionales y la gestión logística influyen positivamente en la gestión de los proyectos de investigación científica financiados con recursos de focam. Así mismo, el Pseudo R cuadrado de Nagelkerke comprueba que las variables independientes explican en un $68,5 \%$ a la variable dependiente del modelo planteado.

Para la prueba de la hipótesis específica 1, de la información de ajuste del planeamiento de la investigación mostrada por SPSS la significancia es 0,000 menor a 0,05, en consecuencia se rechaza la hipótesis nula (Ho) a un nivel de confianza del 95\% y se acepta que las competencias profesionales y la gestión logística influyen positivamente en el planeamiento de la investigación científica financiados con recursos de focam. Así mismo, el Pseudo R cuadrado de Nagelkerke comprueba que las variables independientes explican en un $65,7 \%$ al planeamiento de la investigación del modelo planteado.

Para la prueba de la hipótesis específica 2, de la información de ajuste de la evaluación y aprobación de la investigación mostrada por SPSS la significancia es 0,000 menor a 0,05, en consecuencia, se rechaza la hipótesis nula (Ho) a un nivel de confianza del 95\% y se acepta que las competencias profesionales y la gestión logística influyen positivamente en la evaluación y aprobación de la investigación científica financiados con recursos de focam. Así mismo, el Pseudo R cuadrado de Nagelkerke comprueba que las variables independientes explican en un $60,3 \%$ a la evaluación y aprobación de la investigación del modelo planteado.

Para la prueba de la hipótesis específica 3, de la información de ajuste del planeamiento de la investigación mostrada por SPSS la significancia es 0,000 menor a 0,05, en consecuencia se rechaza la hipótesis nula (Ho) a un nivel de confianza del 95\% y se acepta que las competencias profesionales y la gestión logística influyen positivamente en la organización de 
la investigación científica financiados con recursos de focam. Así mismo, el Pseudo R cuadrado de Nagelkerke comprueba que las variables independientes explican en un $61,4 \%$ a la organización de la investigación del modelo planteado.

Para la prueba de la hipótesis específica 4, de la información de ajuste del planeamiento de la investigación mostrada por SPSS la significancia es 0,000 menor a 0,05, en consecuencia se rechaza la hipótesis nula (Ho) a un nivel de confianza del 95\% y se acepta que las competencias profesionales y la gestión logística influyen positivamente en la implementación de la investigación científica financiados con recursos de Focam. Así mismo, el Pseudo R cuadrado de Nagelkerke comprueba que las variables independientes explican en un $34,1 \%$ a la implementación de la investigación del modelo planteado.

Para la prueba de la hipótesis específica 5, de la información de ajuste del planeamiento de la investigación mostrada por SPSS la significancia es 0,000 menor a 0,05, en consecuencia se rechaza la hipótesis nula (Ho) a un nivel de confianza del 95\% y se acepta que las competencias profesionales y la gestión logística influyen positivamente en la ejecución de la investigación científica financiados con recursos de focam. Así mismo, el Pseudo R cuadrado de Nagelkerke comprueba que las variables independientes explican en un 41,7\% a la ejecución de la investigación del modelo planteado.

Para la prueba de la hipótesis específica 6, de la información de ajuste del planeamiento de la investigación mostrada por SPSS la significancia es 0,000 menor a 0,05, en consecuencia se rechaza la hipótesis nula (Ho) a un nivel de confianza del 95\% y se acepta que las competencias profesionales y la gestión logística influyen positivamente en el control y seguimiento de la investigación científica financiados con recursos de focam. Así mismo, el Pseudo R cuadrado de Nagelkerke comprueba que las variables independientes explican en un $41,2 \%$ al control y seguimiento de la investigación del modelo planteado.

Para la prueba de la hipótesis específica 7, de la información de ajuste del planeamiento de la investigación mostrada por SPSS la significancia es 0,000 menor a 0,05, en consecuencia se rechaza la hipótesis nula (Ho) a un nivel de confianza del $95 \%$ y se acepta que las competencias profesionales y la gestión logística influyen positivamente en el cierre de la investigación científica financiados con recursos de focam. Así mismo, el Pseudo R cuadrado de Nagelkerke comprueba que las variables independientes explican en un 40,5\% al cierre de la investigación del modelo planteado.

Para la prueba de la hipótesis específica 8, de la información de ajuste del planeamiento de la investigación mostrada por SPSS la significancia es 0,000 menor a 0,05, en consecuencia se rechaza la hipótesis nula (Ho) a un nivel de confianza del 95\% y se acepta que las 
competencias profesionales y la gestión logística influyen positivamente en la comunicación de la investigación científica financiados con recursos de Focam. Así mismo, el Pseudo R cuadrado de Nagelkerke comprueba que las variables independientes explican en un $31,2 \%$ a la comunicación de la investigación del modelo planteado.

De los resultados obtenidos de la variable 1 (competencias profesionales), se puede interpretar que cerca del $80 \%$ de los profesionales de ingeniería que tienen un nivel bajo y regular de competencias profesionales, no tienen el nivel de competencias duras y blandas requeridas para realizar una buena gestión de su proyecto de investigación con recursos de Focam. Si el profesional no reúne las competencias duras requeridas para desarrollar un proyecto de investigación es probable que la planificación y su producto (perfil o proyecto de investigación) no estén bien formulado ni consistente con la metodología apropiada para su desarrollo. Además, si el profesional no reúne las competencias blandas necesarias existe la posibilidad de que no pueda cumplir con las etapas del ciclo del proyecto de investigación como son la organización, la implementación, la ejecución y el cierre del proyecto de investigación.

De los resultados obtenidos de la variable 2 (gestión logística), más del 71\% de los profesionales de ingeniería que realizan investigación científica con recursos de focam, perciben un nivel bajo de gestión logística de sus proyectos, lo cual se puede entender que la gestión logística de la gran mayoría de los proyectos de investigación de los profesionales de ingeniería no es eficaz, por lo que influye negativa y significativamente para realizar una buena gestión de los proyectos de investigación, retrasando y afectando directamente las etapas de implementación y ejecución así como a las etapas siguientes de la investigación. Pero se debe indicar que la oficina de logística se encarga de atender a todas las dependencias académicas y administrativas de la institución, lo cual puede ser una de las causas del bajo nivel de gestión logística para atender a los proyectos de investigación. Posiblemente exista alguna relación de influencia entre el grupo del $79 \%$ de profesionales que presentan niveles bajos y regulares de competencias profesionales y la percepción de nivel bajo de gestión logística de sus proyectos de investigación (71.4\%), debido seguramente a que dichos profesionales no realizan correctamente el requerimiento de contratación de bienes y servicios, ni apoyan en el estudio de mercado para realizar y obtener las cotizaciones requeridas por los proyectos.

De los resultados obtenidos de las dimensiones de la variable 2 , se puede interpretar que más del 83,0\% de los profesionales de ingeniería perciben una gestión logística deficiente (nivel bajo y medio) de sus proyectos prácticamente en casi todos los procesos involucrados por las que pasan los proyectos de investigación. Esto afecta directamente la implementación de la investigación, retrasando la adquisición de bienes y servicios requeridos. La deficiencia de la 
gestión logística se puede deber a varias razones, entre ellas relacionadas con los procesos a cumplir que establece las normas y directivas que emite la OSCE lo cual toma tiempo y dedicación, se puede deber al mismo personal administrativo que no cumple con responsabilidad e idoneidad las tareas administrativas que exigen la gestión logística de los proyectos de investigación. Pero también existe la posibilidad de que la deficiencia de la gestión logística de los proyectos de investigación se deba a la misma área usuaria, es decir, los profesionales no cumplen o no ayudan con la información solicitada o requerida para las etapas de requerimiento de contratación y cotización y cuadro comparativo.

De los resultados obtenidos de la variable 3 (gestión de los proyectos de investigación científica), se puede deducir que cerca del $80 \%$ de los profesionales de ingeniera que realizan investigación científica con recursos de focam, llevan a cabo una gestión deficiente (nivel bajo y medio) de los proyectos de investigación científica, y por tanto no logran alcanzar los objetivos y resultados con la calidad deseada, en el tiempo y costos previstos. Se podría deducir que esta gestión deficiente de sus proyectos de investigación está relacionada con el grupo de más o menos del $80 \%$ de profesionales que presentan un nivel deficiente de competencias profesionales (nivel bajo y regular).

De los resultados obtenidos de las dimensiones de la variable 3, se puede interpretar que más del 94\% de los profesionales de ingeniería realizan una gestión deficiente (nivel bajo y regular) de sus proyectos de investigación principalmente en las etapas de evaluación y organización de la investigación. Se puede deducir que este grupo de profesionales de ingeniería no realiza un buen planeamiento de la investigación y difícilmente pasara con buena calificación los criterios de evaluación del proyecto. Cuando el responsable del proyecto no ha realizado una buena organización de la investigación, difícilmente se podrán realizar las actividades y tareas para cumplir con los resultados y objetivos esperados durante la ejecución y seguimiento del proyecto de investigación. Así mismo, más del 65,3\% de los profesionales de ingeniería realizan una gestión deficiente de sus proyectos de investigación en las etapas siguientes de implementación, ejecución, seguimiento y control, cierre y comunicación de la investigación. Y la diferencia que representa un porcentaje menor al 28,7\% (94\% - 65.3\%) de los profesionales de ingeniería, logran superar los inconvenientes y efectuar una buena gestión de sus proyectos hasta la etapa de cierre, ya que ningún profesional investigador logra terminar la gestión con la comunicación de la investigación debido a que posiblemente no se termina de publicar el artículo científico en una revista indizada. 


\section{Referencias}

Aarseth, W., Rolstadas, A. y Andersen, B. (2013). "Managing organizational challenges in global projects", International Journal of Managing Projects in Business, 7 (1), 103 132. Disponible en https://doi.org/10.1108/IJMPB-02-2011-0008.

Alcelay, C. (2014). Manual para la certificación pmp®: cómo aplicar la guía del pmboK® quinta edición en la gestión de proyectos. España: Edita: (C) Fundación Confemetal.

Alpert, S. A. y Hartshorne, R. (2013). An examination of assistant professors project management practices. International Journal of Educational Management, 27 (5), 541 554. Doi: https://doi.org/10.1108/IJEM-1-2012-0023.

Arroyo, R. (2012). Habilidades gerenciales: desarrollo de destrezas, competencias y actitud. Bogotá: Ecoe Ediciones.

Asamblea Estatutaria UNH (2014). Estatuto de la Universidad Nacional de Huancavelica. Huancavelica: Resolución $\mathrm{N}^{\circ}$ 001-2014-AE-UNH.

Bauer, W., Bleck-Neuhaus, J. y Dombois, R. (2010). Desarrollo de proyectos de investigación. Alemania: Universidad de Bremen, Servicio Alemán de Intercambio Académico (DAAD).

Bermúdez, L. y Rodríguez, L. (2013). Investigación en la gestión empresarial. Colombia: Ecoe Ediciones.

Caballero, J. (2015). Factores críticos en los resultados de los proyectos de investigación de convocatorias públicas de un organismo público de investigación (OPI): el caso de la Universitat Politécnica de Valencia. Tesis doctoral. Universidad Politécnica de Valencia.

Carrasco, S. (2013). Metodología de la investigación científica. Perú: editorial San Marcos E.I.R.L.

Castro, M. (2003). El proyecto de investigación y su esquema de elaboración. Caracas: editorial Uyapal.

Concepción, R. (2007). Metodología de gestión de proyectos en las administraciones públicas según ISO 10.006. Tesis doctoral. Universidad de Oviedo.

Conexión Esan (2015). Competencias laborales: Diferencias entre habilidades blandas y duras. Disponible en https://www.esan.edu.pe/apuntesempresariales/2015/06/competencias-laborales-diferencias-entre-habilidades-blandasduras/.

Charry, J. (2009). Factores institucionales y personales relacionados a la capacitación para elaborar proyectos de investigación científica en doctorandos de la Universidad Nacional de Educación. Tesis doctoral. Universidad Nacional Mayor de San Marcos.

Chica, S. (2011). Una mirada a los nuevos enfoques de la gestión pública. Administración \& Desarrollo 39. Bogotá, D.C. Escuela Superior de Administración Pública, ESAP.

Dirección Universitaria de Investigación de la UNH (2014). Reglamento para trabajos de investigación científica y tecnológica para docentes, financiados con recursos de Focam. Huancavelica: Resolución de consejo universitario N$^{\circ}$ 0074-2014-CU-UNH.

EPIE (2017). Diseño curricular de la carrera de Ingeniería Electrónica 2017-2019. Huancavelica: impresiones UNH. 
Estrada, F. M. L., Quiñónez, E. S. y Pantoja, J. P. (2017). El docente universitario como promotor del pensamiento crítico, competencia del investigador. Aula de Encuentro, 19 (2), 58-75.

Fernandes, E. y Guimarães, D. (2013). Proyectos Educativos y Sociales: Planificación, gestión, seguimiento y evaluación. Madrid: Narcea, S. A. de Ediciones.

Gallego, J.L. y Rodríguez, A. (2015). Líneas de investigación sobre Educación Especial en España: un estudio bibliométrico (2006-2010). Revista de Ciencias Sociales, 21 (2), 219233. En http://produccioncientificaluz.org/index.php/rcs/article/view/20003/19936

Gómez, J. (2015). Las competencias profesionales. Revista mexicana de anestesiología, 38 (1), 49-55.

Gómez, A. y Acevedo, J. (2007). La logística moderna en la empresa, volumen 1. La Habana: editora LOGICUBA.

Grolimund, C. (2011). Claves de la Gestión de Proyectos: Gestión eficiente de proyectos y de trabajo en equipo. España: Edita: (C) Fundación Confemetal.

Hernández, R. (2013). Metodología de la Investigación. Disponible en https://es.scribd.com/doc/121347563/Metodologia-de-La-Investigacion-RobertoHernandez-Sampieri.

Hernández, R., Fernández, C., Baptista, P. (2014). Metodología de la investigación. México: Mcgraw-Hill / Interamericana Editores, S.A. de C.V.

Herrera, C. (2016). Estrategias investigativas y su influencia en la elaboración del proyecto de investigación científica. Caso: estudiantes de la facultad de Ciencias políticas y Administrativas de la Universidad Nacional de Chimborazo. Riobamba, Ecuador, Tesis doctoral. Universidad Nacional Mayor de San Marcos.

MOF-UNH. (2014). Manual de organización y funciones de la Universidad Nacional de Huancavelica. Huancavelica: Impresiones UNH.

Morris, P.W.G. (2010). Research and the future of project management. International Journal of Managing Projects in Business, 3 (1), 139-146. Doi: https://doi.org/10.1108/17538371011014080.

Ñaupas, H., Mejía, E., Novoa, E., Villagómez, A. (2011). Metodología de la investigación científica y asesoramiento de tesis. Perú: Centro de producción editorial e imprenta de la UNMSM.

OSCE-L. (2014). Ley de las contrataciones del estado: ley $\mathrm{N}^{\circ}$ 30225. Disponible en https://www.mef.gob.pe/es/normatividad-externa/13832-05-rdn-006-2012-piptransporte-estudios-en-paquete-mod-anexo-snip-09-10-y-16-2-2-final-531/file.

OSCE-R. (2015). Reglamento de la ley de contrataciones del estado: ley No 30225. Disponible en http://portal.osce.gob.pe/osce/sites/default/files/Documentos/legislacion/ley/Reglamen to\%20de\%20la\%20Ley\%20N\%C2\%BA\%2030225_0.pdf.

Ram, J. y Ronggui, D. (2018). Research and development projects: An empirical investigation of project managers' traits. International Journal of Managing Projects in Business. Doi: https://doi.org/10.1108/IJMPB-03-2017-0032.

Ramón, J., García, J. y Lamarca, I. (2007). Gestión de proyectos informáticos: métodos, herramientas y casos. Barcelona: editorial UOC. 
RegFocamDUI. (2012). Reglamento para trabajos de investigación científica y tecnológica para docentes, financiado con recursos focam. Huancavelica: Impresiones UNH.

Rodríguez, A. (2005). Research on special education needs: what and how to investigate in special education, Electronic Journal of Research in Educational Psychology, 5, 3 (1), 97-112. En http://www.investigacionpsicopedagogica.org/revista/new/english/ContadorArticulo.php?41

Rodríguez, A. y Caurcel, M. J. (2019). Nota de investigación. Aproximación cualitativa al escudriño en psicología educativa. Propósitos y Representaciones, 7 (1). En http://revistas.usil.edu.pe/index.php/pyr/issue/view/23

Rodríguez, E., Scavuzzo, J., Taborda, A. y Buthet, C. (2013). Metodología integral de evaluación de proyectos sociales. Argentina: editorial Brujas.

ROF-UNH (2014). Reglamento de organización y funciones de la Universidad Nacional de Huancavelica. Huancavelica: Impresiones UNH.

Sáenz, A. R. (2013). El éxito de la gestión de proyectos. Un nuevo enfoque entre lo tradicional y lo dinámico. Tesis doctoral. Universidad Ramon Llull.

Stracuzzi, S. y Pestana, F. (2012). Metodología de la investigación cuantitativa. Venezuela: Fondo Editorial de la Universidad Pedagógica Experimental Libertador (FEDUPEL). La editorial pedagógica de Venezuela.

Toro, F. J. y Bernate, G. (2013). Gerencia de proyectos: aplicaciones en salud. Colombia: ECOE Ediciones.

UNH (2016a). Formulación del requerimiento y procedimiento para la contratación de bienes y servicios: Directiva $N^{\circ}$ 01-2016-UNH/OL. Huancavelica: Impresiones UNH.

UNH (2016b). Normas y procedimientos para la contratación de bienes y servicios menores o iguales a 8 UIT en la UNH: Directiva $\mathrm{N}^{\circ}$ 07-2016-DPP-R/UNH. Huancavelica: Impresiones UNH.

UNH-ME (2014). Modelo educativo de la UNH 2014. Huancavelica: Vicerrectorado Académico de la UNH.

UNH-PEI (2016). Plan Estratégico Institucional, PEI 2016-2020. Huancavelica: Dirección Universitaria de Planificación y Presupuesto de la UNH.

Vicepresidencia Académica de la comisión de orden y gestión de la UNH (2012). Reglamento para trabajos de investigación científica y tecnológica para docentes, financiad con recursos de Focam. Huancavelica: Resolución de consejo universitario $\mathrm{N}^{\circ}$ 0598-2012COGUNH-ANR.

Vicerrectorado de Investigación de la UNH (2016). Reglamento para Elaboración de Proyectos, Ejecución e Informe Final de Investigación Científica, Tecnológica y de Innovación Subvencionados con Recursos Del "Fondo De Desarrollo Socioeconómico del proyecto camisea". Huancavelica: Resolución de consejo universitario $\mathrm{N}^{\circ}$ 02842016-CU-UNH.

Villegas, K. (2006). Concepto de logística: Capítulo 11. Gestión logística. Disponible en http://www.mailxmail.com/curso-concepto-logistica/gestion-logistica. 